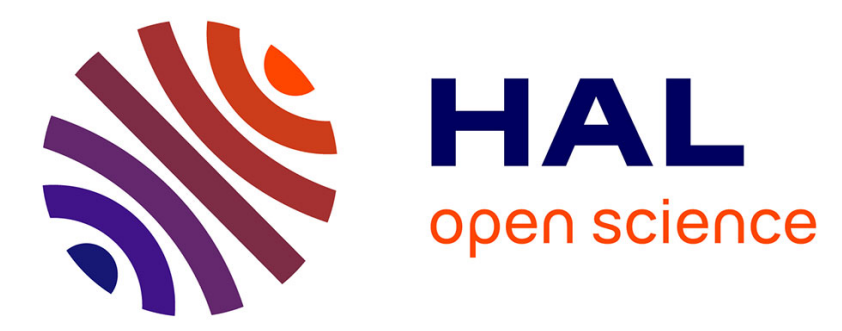

\title{
Discussion on the Structure Stability and the Luminescence Switch under Irradiation of a Ce-Doped Elpasolite Compound.
}

Lucile Cornu, Manuel Gaudon, Philippe Veber, Antoine Villesuzanne, Stanislav Pechev, Alain Garcia, Veronique Jubera

\section{To cite this version:}

Lucile Cornu, Manuel Gaudon, Philippe Veber, Antoine Villesuzanne, Stanislav Pechev, et al.. Discussion on the Structure Stability and the Luminescence Switch under Irradiation of a Ce-Doped Elpasolite Compound.. Chemistry - A European Journal, 2015, 21 (13), pp.5242-5251. hal-01135935

\author{
HAL Id: hal-01135935 \\ https://hal.science/hal-01135935
}

Submitted on 22 Jan 2021

HAL is a multi-disciplinary open access archive for the deposit and dissemination of scientific research documents, whether they are published or not. The documents may come from teaching and research institutions in France or abroad, or from public or private research centers.
L'archive ouverte pluridisciplinaire HAL, est destinée au dépôt et à la diffusion de documents scientifiques de niveau recherche, publiés ou non, émanant des établissements d'enseignement et de recherche français ou étrangers, des laboratoires publics ou privés. 


\title{
Discussion on the Structure Stability and the Luminescence Switch under Irradiation of a Ce-Doped Elpasolite Compound
}

\author{
Lucile Cornu, ${ }^{[a, b]}$ Manuel Gaudon, ${ }^{*[a, b]}$ Philippe Veber, ${ }^{[a, b]}$ Antoine Villesuzanne, ${ }^{[a, b, c]}$ \\ Stanilas Pechev, ${ }^{[a, b]}$ Alain Garcia, ${ }^{[a, b]}$ and Véronique Jubera* ${ }^{* a, b]}$
}

Abstract: Ce-doped $\mathrm{Rb}_{2} \mathrm{KInF}_{6}$ elpasolite has the potential for tunable luminescence due to an unusual reversible redox process between the cerium and indium cations. Coupled with a deep understanding of the luminescence properties, XRD analysis and DFT calculations are used to locate the doping elements in the host lattice. The origin explanation of the charge-transfer mechanism that causes a decrease or increase in the blue-green cerium emission in opposition to the red indium emission is discussed regarding the crystallographic structure, the connection of the metallic cations and their equilibrium valence. Still detectable after nineteen years, the optical contrast created under irradiation makes this material a good candidate as photosensor for data storage.

\section{Introduction}

Discovered in $1883,{ }^{[1]}$ in El Paso county, the so-called elpasolite phases are described by the general formula $\mathrm{A}_{2} \mathrm{BMX}_{6}{ }^{[2]} \mathrm{A}$ and $B$ belong to the alkali group, $M$ is a transition metal or a rareearth element, and $\mathrm{X}$ is an halogen. The properties obtained for various compositions are the subject of numerous studies. ${ }^{[3-7]}$ Transition-metal- or rare-earth-doped elpasolite matrices are used in several emitting systems, such as laser devices, ${ }^{[8-10]}$ light-emitting diodes, quantum cutters, ${ }^{[11]}$ crystal scintillators, ${ }^{[12,13]}$ and neutron and gamma detectors. ${ }^{[14]}$ In addition, these matrices are used in medical radiation detection apparatuses, ${ }^{[15]}$ and in data storage devices. ${ }^{[16]}$ On the one hand, this large field of applications is because of the wide range of compositions that crystallize in the elpasolite framework, on the other hand, the structural configuration of this matrix, which offers different cationic substitution sites for the doping element, makes possible significant modification of the luminescent properties. The elpasolite material belongs to the $F m \overline{3} m$ space group and can be described as a double perovskite with cationic ordering. Elpasolite is also called a B-site rock-salt perovskite with $\mathrm{A}_{2} \mathrm{BMX}_{6}$ composition. ${ }^{[17]}$ The $\mathrm{A}$ cation is located in the central cuboctahedral site ( $8 c$ Wyckoff position). The $B$

[a] Dr. L. Cornu, Dr. M. Gaudon, Dr. P. Veber, Dr. A. Villesuzanne, Dr. S. Pechev, Dr. A. Garcia, Dr. V. Jubera

CNRS, ICMCB, UPR 9048, 33600 Pessac (France)

E mail:gaudon@icmcb bordeaux.cnrs.fr veronique.jubera@u bordeaux.fr

[b] Dr. L. Cornu, Dr. M. Gaudon, Dr. P. Veber, Dr. A. Villesuzanne, Dr. S. Pechev, Dr. A. Garcia, Dr. V. Jubera

Univ. Bordeaux, ICMCB, UPR 9048, 33600 Pessac (France)

[c] Dr. A. Villesuzanne

IREET, University of Bolton, Bolton, BL3 $5 A B$ (UK) alkali cation and the $\mathrm{M}$ transition-metal/rare-earth element regularly alternate along the unit cell axis in an octahedral site ( $4 b$ and $4 a$ positions).

The Ce-doped $\mathrm{Rb}_{2} \mathrm{KInF}_{6}$ elpasolite was previously studied by Chaminade et al. ${ }^{[13]}$ These authors announced that the $\mathrm{Ce}^{3+}$ doping element was located in three sites. The primary emission at $\lambda=480 \mathrm{~nm}$ reflects the normal substitution of the trivalent element $\mathrm{In}^{3+}$ by $\mathrm{Ce}^{3+}$ in the regular octahedron. Two minor emissions at $\lambda=320$ and $424 \mathrm{~nm}$ were attributed to trivalent cerium in the rubidium and potassium crystallographic sites. In addition, under UV irradiation, the authors observed a redox phenomenon between the $\mathrm{Ce} / \mathrm{In}$ ions that led to a reversible luminescence process based on the following equilibrium: $2 \mathrm{Ce}^{3+}+\mathrm{In}^{3+} \rightleftarrows 2 \mathrm{Ce}^{4+}+\mathrm{In}^{+}$. To the best of our knowledge, with the exception of this fluoride compound, this type of redox property has only been observed in sodium borate glass under gamma irradiation, ${ }^{[18]}$ and is a promising feature for data storage applications or photoluminescent detectors. Because such optical behavior is rare, in this article, we propose to explain the efficiency of the redox process from a structural perspective. In the first part, the possibility of predicting the structural stability and the atomic positioning is developed from ab initio calculations (density functional theory); the cationic distribution (between $\mathrm{Rb}^{+}, \mathrm{K}^{+}, \mathrm{In}^{3+}$, and $\mathrm{Ce}^{3+}$ ) and the $\mathrm{F}^{-}$ion disorder, which leads to octahedral tilts, is discussed in terms of Rietveld refinements. A new $\mathrm{Ce}^{3+}$ localization is also discussed from optical measurements. In the last section, the redox phenomenon between In $^{\text {III }}$ and Ce $\mathrm{Ce}^{\text {III }}$ photoactivated under UV irradiation is accurately investigated, and lastly, a full interpretation of this phenomenon is proposed. 


\section{Results and Discussion}

Structural characterization and ab initio atomic positioning

\section{Structural characterization, $F$ ion positions}

A single crystal was selected after the Bridgman crystal growth process and crushed. The corresponding X-ray diffraction analyses confirm the formation of a pure elpasolite phase; all of the diffraction peaks belong to the $\mathrm{Rb}_{2} \mathrm{KInF}_{6}$ phase (JCPDS file no. 00-048-1754) with cubic form ( $F m \overline{3} m$ space group) (Figure $1 \mathrm{a}$ ).
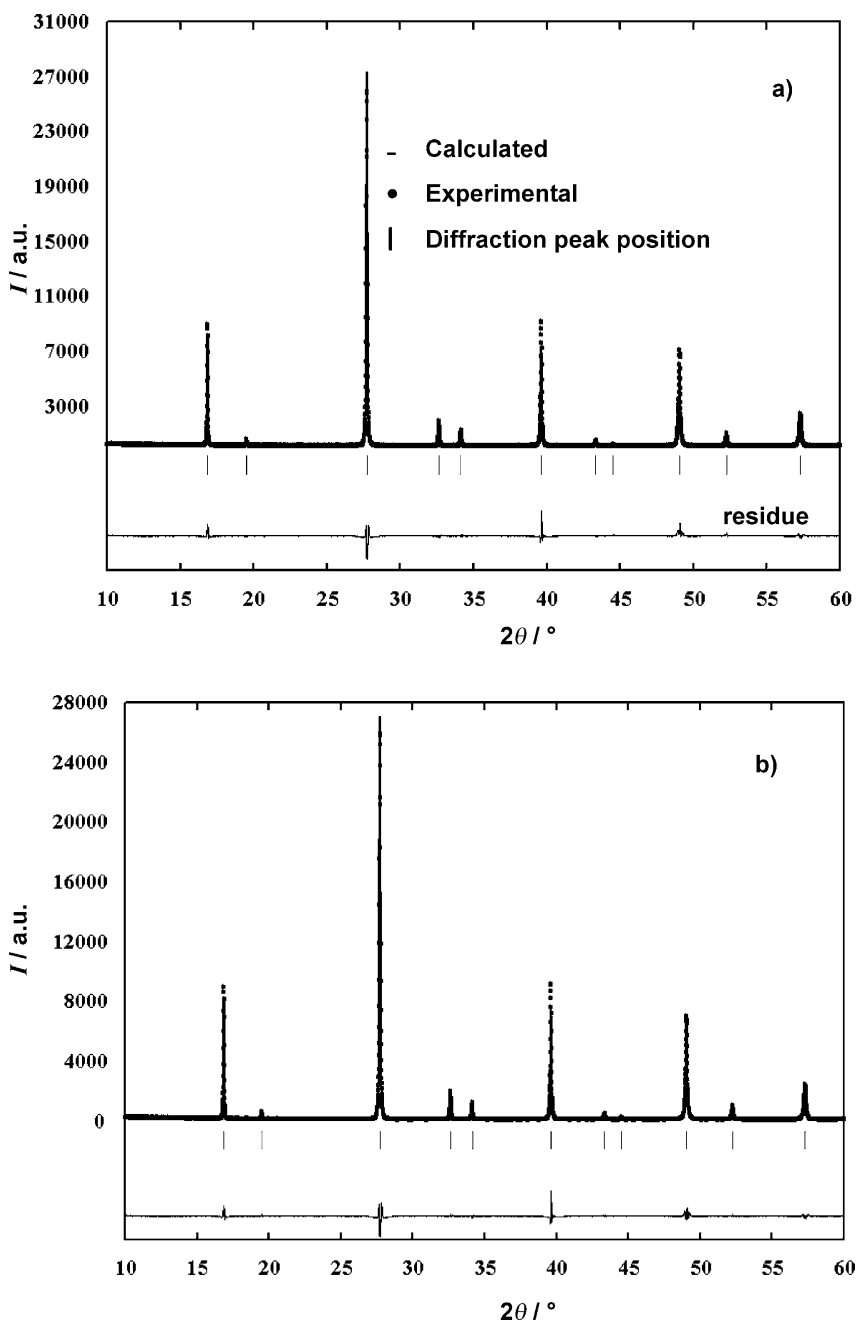

Figure 1. Refined diffractograms for $\mathrm{Rb}_{2} \mathrm{KInF}_{6} / \mathrm{Ce} 2 \%$. a) Refined with $\mathrm{F}$ in 24 e positions and $\mathrm{b}$ ) refined with $\mathrm{F}$ in $96 j$ positions.

The obtaining of a pure Ce-doped $\mathrm{Rb}_{2} \mathrm{KInF}_{6}$ compound opened the window for an accurate structural investigation of this compound. Rietveld refinement was first performed based on an "ordered" or "regular" cubic structure, for which the pattern representation of the structural framework is reported in Figure $\mathrm{S} 1 \mathrm{a}$ in the Supporting Information. In such a regular cubic form, the fluorine ions occupy the $24 e$ Wyckoff positions located on the cell edges, that is, on the axis of the $\mathrm{In}^{3+}-\mathrm{K}^{+}$ cations. The Rietveld refinement pattern is reported in
Figure $1 \mathrm{a}$; the refined structural parameters, the isotropic displacement factors, and the reliability coefficients are reported in Table 1.

\begin{tabular}{|c|c|c|c|c|}
\hline $\begin{array}{l}\text { Powder refinement } \\
\text { Fluorine atom } \\
\text { Wyckoff positions }\end{array}$ & $\begin{array}{l}\text { ushed singl } \\
a[\AA]\end{array}$ & $\begin{array}{l}\text { ystal) } \\
x_{F}\end{array}$ & $z_{\mathrm{F}}$ & $B_{\text {iso }}\left[\AA^{2}\right]^{[b]}$ \\
\hline $24 e$ & 9.0934 & 0.2219 & 0 & $\begin{array}{r}\mathrm{Rb} 2.49 \\
\mathrm{~K} 1.33 \\
\operatorname{In} 0.51 \\
\mathrm{~F} 6.73\end{array}$ \\
\hline \multirow[t]{3}{*}{$96 j$} & 9.0933 & 0.2223 & 0.0457 & $\begin{array}{r}\mathrm{Rb} 2.63 \\
\mathrm{~K} 1.29 \\
\text { In } 0.57 \\
\mathrm{~F} 1.73\end{array}$ \\
\hline & \multicolumn{2}{|c|}{ reliability factors ${ }^{[a]}$} & & \\
\hline & $\mathrm{Rp}$ & $\mathrm{Chi}^{2}$ & \multicolumn{2}{|c|}{ Bragg $R$ factor } \\
\hline $24 e$ & 11.1 & 2.89 & \multicolumn{2}{|l|}{6.20} \\
\hline $96 j$ & 10.6 & 2.72 & \multicolumn{2}{|l|}{4.84} \\
\hline \multicolumn{5}{|c|}{ Single crystal refinement } \\
\hline $\begin{array}{l}\text { Fluorine atom } \\
\text { Wyckoff positions }\end{array}$ & $a[\AA]$ & $x_{\mathrm{F}}$ & $z_{\mathrm{F}}$ & $B_{\text {iso }}\left[\AA^{2}\right]^{[b]}$ \\
\hline \multirow[t]{4}{*}{$24 e$} & $9.0835(5)$ & 0.2408 & 0 & $\mathrm{Rb} 3.13$ \\
\hline & & & & K 1.33 \\
\hline & & & & $\ln 1.43$ \\
\hline & & & & F 7.45 \\
\hline \multirow[t]{6}{*}{$96 j$} & $9.0835(5)$ & 0.2228 & 0.0378 & $\mathrm{Rb} 3.11$ \\
\hline & & & & K 1.78 \\
\hline & & & & $\ln 1.41$ \\
\hline & & & & F 3.75 \\
\hline & \multicolumn{4}{|c|}{ reliability factors } \\
\hline & $R 1$ & $w R 2$ & GooF & \\
\hline $24 e$ & 2.40 & 4.42 & 1.17 & \\
\hline $96 j$ & 2.17 & 3.16 & 1.15 & \\
\hline
\end{tabular}

[a] With all non excluded and not corrected for background. [b] For the single crystal refinement $B_{\text {iso }}=8 \pi^{2} \times U_{\text {eq. }}$.

The refinement leads to acceptable reliability factors but to a very high isotropic displacement factor for the fluorine ions. Furthermore, the cation-anion distances extracted from the refinement allow the calculation of the valences for different ions from the Brown and Altermatt equation [Eq. (1)]. ${ }^{[19]}$

$V_{i}=\sum_{j}^{i} \exp \left(\frac{r_{0}^{\prime} \quad r_{i j}}{B}\right)$

where $V_{i}$ is the valence of the cation $i, r_{0}^{\prime}$ and $B$ are empirically determined parameters, and $r_{i j}$ is the distance between the cations $i$ and $j$.

For this calculation, the impact of cerium doping on the bond lengths was considered to be negligible, so the calculation was performed based on a pure $\mathrm{Rb}_{2} \mathrm{KInF}_{6}$ composition. Furthermore, the $r_{0}$ parameters tabulated by Brown and Altermatt, ${ }^{[19]}$ were used for the $\mathrm{In} \mathrm{F}$ and $\mathrm{K} \mathrm{F}$ bonds with $r_{0}$ equal to 1.792 and $1.992 \AA$, respectively, whereas the $r_{0}$ parameter for 
the $\mathrm{Rb} F$ bond was extracted from our own calculation for the fluoride $\mathrm{RbF}$; the calculated $r_{0}$ is $2.12 \AA$. The valence calculated for each cation and the valence residues (differences between the experimental and theoretical valences) are reported in Table 2.

\begin{tabular}{|c|c|c|c|c|}
\hline Wyckoff position & bond & distances $[\AA]]$ & valences & valence residue \\
\hline \multicolumn{5}{|c|}{ calculation on powder refinement } \\
\hline \multirow[t]{3}{*}{$24 e$} & In $\mathrm{F}$ & $2.018(7)$ & $3.2(4)$ & $0.2(4)$ \\
\hline & $\mathrm{K} \mathrm{F}$ & $2.528(0)$ & 1.4(1) & $0.4(1)$ \\
\hline & $\mathrm{Rb} F$ & $3.225(1)$ & $0.5(2)$ & $0.4(8)$ \\
\hline \multirow[t]{4}{*}{$96 j$} & In $\mathrm{F}$ & $2.063(7)$ & $2.8(8)$ & $0.1(2)$ \\
\hline & K F & $2.559(2)$ & $1.3(0)$ & $0.3(0)$ \\
\hline & $\mathrm{Rb} \mathrm{F}_{1}$ & $2.946(7)$ & $0.7(4)$ & $0.2(6)$ \\
\hline & $\mathrm{Rb} \mathrm{F}_{2}$ & $3.530(1)$ & & \\
\hline \multicolumn{5}{|c|}{ calculation on single crystal refinement } \\
\hline \multirow[t]{3}{*}{$24 e$} & $\ln \mathrm{F}$ & $2.035(4)$ & $3.1(1)$ & $0.1(1)$ \\
\hline & $\mathrm{K} \mathrm{F}$ & $2.506(4)$ & $1.4(1)$ & $0.4(1)$ \\
\hline & $\mathrm{Rb} F$ & $3.2201(4)$ & $0.5(2)$ & $0.4(8)$ \\
\hline \multirow[t]{4}{*}{$96 j$} & In $\mathrm{F}$ & $2.053(5)$ & $2.9(6)$ & $0.0(4)$ \\
\hline & $\mathrm{K} \mathrm{F}$ & $2.541(5)$ & $1.3(6)$ & $0.3(6)$ \\
\hline & $\mathrm{Rb} \mathrm{F}_{1}$ & $2.989(10)$ & $0.6(8)$ & $0.3(2)$ \\
\hline & $\mathrm{Rb} \mathrm{F}$ & $3.472(10)$ & & \\
\hline
\end{tabular}

The experimental valence of the $\mathrm{Rb}^{+}$ion is seen to be much smaller than the theoretical value, whereas the valences of the $\mathrm{In}^{3+}$ and $\mathrm{K}^{+}$ions are much larger than the theoretical values. This observation can be explained by the Goldschmidt, or tolerance, factor $t$ of our elpasolite phase. This tolerance factor is defined by the same equation used for double perovskites; ${ }^{[3]}$ the value of 0.89 is based on the theoretical $R b F$, In $F$, and $\mathrm{K} F$ bond lengths (the values that perfectly satisfied the ion valences from the valence law) in regular cuboctahedral and octahedral environments, respectively. The tolerance factor, which is significantly less than 1 , indicates that the cations at the $B$ sites are too large relative to the cation at the $A$ site. This difference in size creates strain, which should be responsible for structural distortion similar to that observed in simple perovskite systems. Indeed, the $\mathrm{Rb}_{2} \mathrm{KInF}_{6}$ elpasolite phase is known to exhibit phase transitions associated with the tilt of the octahedral sites as the temperature decreases; at low temperatures, these transitions lead to distorted phases (tetragonal and then monoclinic systems). ${ }^{[20]}$ When the tolerance factor is less than 1, to a first approximation, we consider that the simplest way to "stabilize" the structure is to distort the $\mathrm{Rb}^{+}$ cuboctahedral site whereas maintaining the symmetry of the octahedral sites. As is well predicted by the valence model, the distortion of the $\mathrm{Rb}^{+}$sites would lead to an increase in the valence of the Rb cation. ${ }^{[21]}$ Such a distortion of the cuboctahedron can be achieved by tilting the $\mathrm{KF}_{6}$ and $\mathrm{InF}_{6}$ octahedra. Hence, in this basis, the fluorine ions can be thought to move from the 24 e positions to the $96 j$ positions; this is the simplest anionic disorder that allows rotation of the octahedral sites. In this way, the rotation can involve fluorine disorder between the four sub-positions (96j Wyckoff positions), and the average cubic structure is predicted to be maintained because opposite octahedral tilts occur with the same probability, as illustrated in Figures S1 b and S1 c in the Supporting Information.

At this point, we can explain the poor experimental ion valences and the fluorine isotropic factor found by the Rietveld refinement in terms of the statistical disorder of fluorine ions in the $96 j$ sub-positions. The $96 j$ positions are characterized relative to the $24 e$ positions by a decentering from the $\mathrm{K}-\mathrm{In}$ axis with an intensity of $z_{\mathrm{F}}$ (amplitude of shift from the K-In axis). Before attempting to refine the structure with fluorine in the $96 j$ positions with coordinates $\left(x_{F}, x_{F} z_{F}\right)$, the $z_{F}$ value must be chosen for use in the first iteration of the refinement procedure. In such a disordered elpasolite structure, the distances between the cations and anions are linked to the cell parameters and the fluorine coordinates $x_{\mathrm{F}}$ and $z_{\mathrm{F}}$ through the following Equations (2):

$$
\begin{aligned}
& d_{\mathrm{In}-\mathrm{F}}=a \sqrt{ }\left(z_{\mathrm{F}}^{2}+x_{\mathrm{F}}^{2}\right) \\
& \left.d_{\mathrm{K}-\mathrm{F}}=a \sqrt{(0.5} \quad z_{\mathrm{F}}\right)^{2}+x_{\mathrm{F}}^{2} \\
& d_{\mathrm{Rb}-\mathrm{F} 1}=\sqrt{\left(\left(z_{\mathrm{F}} a\right)^{2}+\left(a^{\sqrt{ } 2 / 4}\right)^{2} 2\left(z_{\mathrm{F}} a\right)\left(a^{\sqrt{ } 2 / 4}\right) \cos (45)\right)^{2}} \\
& d_{\mathrm{Rb}-\mathrm{F} 2}=\sqrt{\left(\left(z_{\mathrm{F}} a\right)^{2}+\left(a^{\sqrt{ } 2 / 4}\right)^{2} 2\left(z_{\mathrm{F}} a\right)\left(a^{\sqrt{ } 2} / 4\right) \cos (135)\right)^{2}}
\end{aligned}
$$

The octahedral sites are composed of six equivalent distances and the distorted 12-coordinated site by six distances $d_{\mathrm{Rb}-\mathrm{F} 1}$ and six distances $d_{\mathrm{Rb}-\mathrm{F} 2}$. Furthermore, the valences of the cations can be calculated from the values of these distances. Then, considering that the distances must fit the theoretical valence for each ion, the system of three equations where $a$, $x_{\mathrm{F}}$, and $z_{\mathrm{F}}$ are the unknown variables can be resolved by using "a least-squares method" applied to the sum of the valence residuals. This ab initio calculation of the $\mathrm{Rb}_{2} \mathrm{KInF}_{6}$ matrix gives the following values: $a=9.0467 \AA, x_{\mathrm{F}}=0.2151$, and $z_{\mathrm{F}}=0.0706$. These values are the input values used to start the new refinement of the elpasolite phase in terms of the disorder of the fluorine ions.

It is worth noting that the previous calculation can be extended to any composition that crystallizes with the elpasolite structure (or even a simple perovskite structure) by arbitrarily changing some ions (i.e., changing the $r_{0}^{\prime}$ value considered and/or the target valence). This ab initio model can easily be achieved and directly links the tolerance factor and the positions of the fluorine ions (or other anions) by considering the tolerance value to be the occurrence of and intensity of tilt in the octahedral sites considered. This model is presented here as a pertinent digression for the simplest systems, which can be considered, that is, the $A B X_{3}$ simple perovskites. Simple perovskite phases can be viewed as special cases of elpasolite or 
double perovskite systems, in which the $B$ and $B^{\prime}$ cations are a single cation and $x_{\mathrm{F}}=0.5$. Both the valence residual after minimization and the corresponding $z_{\mathrm{F}}$ values predicted by our model as a function of the tolerance factor in perovskite are reported in Figures $2 \mathrm{a}$ and $\mathrm{b}$, respectively.
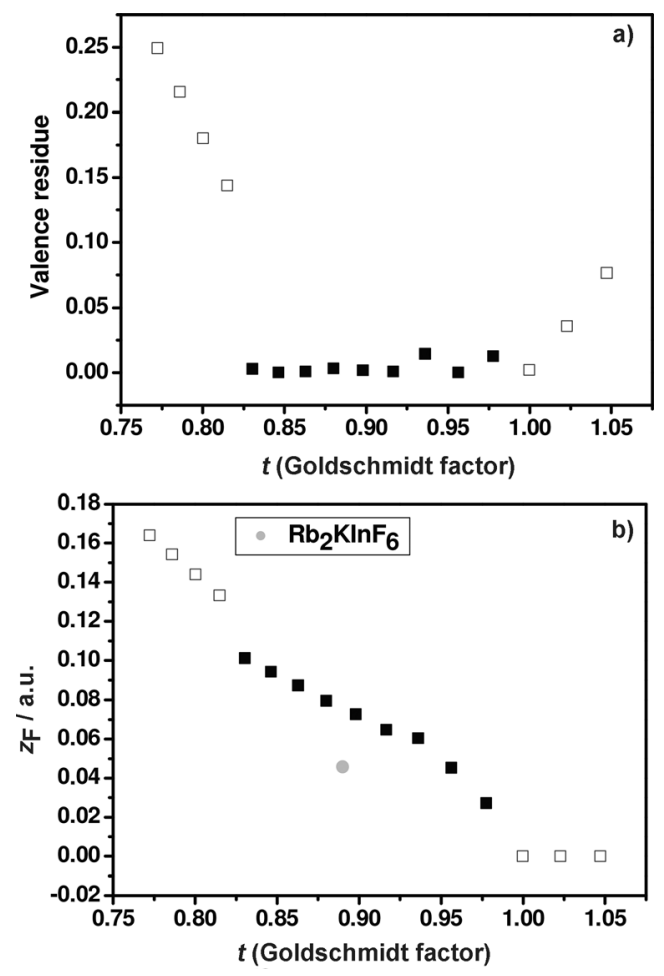

Figure 2. Evolution function of $t$ (tolerance factor) of a) the valence residues and $b$ ) the flour coordinate $z_{\mathrm{F}}$ for the perovskite phases. Full black squares indicate the range of the model validity.

The valence residual curve shows that the calculation is robust for a tolerance factor in the range $0.8<t<1$. Indeed, in this range, the valence residual remains very low; this behavior emphasizes that the octahedral tilts allowed in this range. stabilize the structure (i.e., lead to satisfactory valences for each ion). For extremely low $t$ factors, the calculation shows a sharp increase in the valence residual, indicating that octahedral tilts cannot compensate for the mismatch in the radii of the $B$ and $A$ ions. In the same way, because tilts of the octahedral sites do not adequately relax the strains inside the structure with tolerance factors greater than 1, the net value of the valence residuals also sharply increases in this range and increases the tolerance factor. For such perovskite-type structures, the relaxation occurs through the decentering of ions, such as in $\mathrm{BaTiO}_{3}$ (titanium out-of-center distortion). Regardless, in the validity domain of our model, that is, in the $0.8<t<1$ range, a nearlinear increase of the $z_{\mathrm{F}}$ value is obtained as the $t$ factor decreases. This behavior shows that this simple model is sufficient to predict the increase in the amplitude of the fluorine disorder whereas the structure exhibits the more important mismatch in the radii of the $A$ and $B$ cations.

For fluorine ions in the $96 j$ positions, with an occupancy of 0.25 , the Rietveld refinement results are illustrated in Figure $1 \mathrm{~b}$ and the refined parameters are reported in Table 1. First, in comparison with the refinement process performed for the fluorine ions in the $24 \mathrm{e}$ position, the $96 j$ refinement leads to better reliability factors, even if the decrease in the reliability factors is not drastic. Secondly, the isotropic displacement factor associated with the fluorine ion is now approximately $1.7 \AA^{2}$. Thus, the isotropic displacement factor of the $F^{-}$ions is now relevant in contrast to the isotropic displacement factor observed in the previous procedure $\left(6.7 \AA^{2}\right)$. This observation clearly indicates the improvement of the refinement when anionic disorder is considered. Lastly, the different ion valences extracted from the various bond lengths determined from the last refinement process, all show an improvement; they are all closer to the theoretical values. Without a doubt, consideration of the anionic disorder can be concluded to improve the structural refinement. Nevertheless, this anionic disorder does not seem to be sufficient to lead to a perfect recovery of the theoretical and the experimental distances (or valences). One possible explanation is that tilt of the octahedral sites is not the only way to allow relaxation of the strain; for instance, a slight out-of-center displacement of the $\mathrm{Rb}^{+}$ion may also induce an increase in the valence of the $\mathrm{Rb}$ ion. This displacement can explain the position of the point for the $\mathrm{Rb}_{2} \mathrm{KInF}_{6}$ matrix in Figure $2 \mathrm{~b}$. The gap between the calculated values and the refined ones for the elpasolite matrix is correlated with the fact that the anionic disorder cannot be the only parameter that stabilizes the structure.

In order to validate the methodology applied on powder, single-crystal acquisition was performed. The refined parameters are listed in Table 1 as well as the recalculated valences (Table 2). The location of the fluorine anions on the $96 j$ positions converges as well as previously to more acceptable oxidation states and lower reliability factors. All data concerning single-crystal refinements performed on both hypotheses are reported in the Supporting Information Tables S1a-4a and $\mathrm{S} 1 \mathrm{~b}-4 \mathrm{~b}$ for the $24 e$ and $96 j$ fluorine positions, respectively.

\section{First-principles calculations}

Calculations within the density functional theory (DFT), as implemented in the VASP code, ${ }^{[22-24]}$ were performed to identify the preferential substitution sites for the doping $\mathrm{Ce}^{3+}$ ion. Indeed, this doping ion introduced in small quantity (2 mol\%) is obviously non localizable by X-ray diffraction. Four structural models (labeled hereafter $A$ to $D$ ) were considered for the $\mathrm{Ce}^{3+}$ -to- $\mathrm{In}^{3+}$ substitution, all within a $2 a \times 2 b \times c$ 80-atoms supercell (i.e., $\mathrm{Rb}_{2} \mathrm{Kln}_{0.875} \mathrm{Ce}_{0.125} \mathrm{~F}_{6}$ ): A) the $\mathrm{Ce}^{3+}$ ion is placed in the $\mathrm{In}^{3+}$ site (the structural composition with each site isolated into bracket is then: $\left.\left.\left[\mathrm{Rb}_{2}\right][\mathrm{K}]\left[\mathrm{In}_{0.875} \mathrm{Ce}_{0.125}\right]\left[\mathrm{F}_{6}\right]\right), \mathrm{B}\right)$ the $\mathrm{Ce}^{3+}$ insertion takes place at the $\mathrm{K}^{+}$site and is thus coupled to a $\mathrm{K}^{+}$for $\mathrm{In}^{3+}$ substitution at an indium site $\left(\left[\mathrm{Rb}_{2}\right]\left[\mathrm{K}_{0.875} \mathrm{Ce}_{0.125}\right]\left[\mathrm{In}_{0.875} \mathrm{~K}_{0.125}\right]\left[\mathrm{F}_{6}\right]\right)$, C) similar scheme with $\mathrm{Ce}^{3+}$ inserted at the $\mathrm{Rb}^{+}$site $\left(\left[\mathrm{Rb}_{1.875} \mathrm{Ce}_{0.125}\right][\mathrm{K}]\left[\mathrm{In}_{0.875} \mathrm{Rb}_{0.125}\right]\left[\mathrm{F}_{6}\right]\right)$, and $\left.\mathrm{D}\right)$ involves three sites: $\left[\mathrm{Rb}_{1.875} \mathrm{Ce}_{0.125}\right]\left[\mathrm{K}_{0.875} \mathrm{Rb}_{0.125}\right]\left[\mathrm{In}_{0.875} \mathrm{~K}_{0.125}\right]\left[\mathrm{F}_{6}\right]$.

In each case, the substitution sites are located at the closest possible position in the unit cell, and a full relaxation of the atomic positions (with cell parameters fixed to the experimen- 
tal values) was achieved according to a conjugate-gradient algorithm. The relaxation was pushed beyond hundred steps in each case, in order to ensure full convergence toward the equilibrium structures, and to overcome metastable structures (more precisely, energy plateaus). Note that the use of a $2 a \times$ $2 b \times 2 c$ supercell would have only permitted a limited increase of the minimum $\mathrm{Ce} \mathrm{Ce}$ distance, and a relative change in the number of valence electrons (per cell) lower than $0.1 \%$, for a huge cost in computational time.

The DFT calculation results are given in Table 3. The valence shell electronic energies $\left(\left[\mathrm{eV} \mathrm{cell}^{-1}\right]\right)$ show that model $\mathrm{A}$ leads to the most stable structure. The highest probability is of sub-

Table 3. DFT models, energy, and coordination number of the $\mathrm{Ce}$ ion, max and min distance values, and cerium valence.

\begin{tabular}{|lllll|} 
Model & $\begin{array}{l}\text { Energy } \\
{\left[\mathrm{EV}_{\text {Cell }}{ }^{1}\right]}\end{array}$ & $\begin{array}{l}\text { Coordination } \\
\text { number }\end{array}$ & $\begin{array}{l}d_{\text {Ce F } \min \rightarrow} \\
d_{\text {Ce F } \max [\AA]}\end{array}$ & $\begin{array}{l}\text { Ce } \\
\text { valence }\end{array}$ \\
\hline A & 361.36 & 6 & $2.25 \rightarrow 2.27$ & 3.36 \\
B & 358.46 & 6 & $2.15 \rightarrow 2.38$ & 2.99 \\
C & 358.81 & & & \\
D & 358.93 & 8 & $2.19 \rightarrow 2.60$ & 2.63 \\
\hline
\end{tabular}

stituting $\mathrm{Ce}^{3+}$ for $\mathrm{In}^{3+}$ with no internal side-substitutions involving other neighbor sites. The isovalence of the two ions obviously plays a role in the preference for model A. The relaxed structure shows that the coordination sphere for $\mathrm{Ce}^{3+}$ is a near regular octahedron (the six $\mathrm{Ce} F$ distances lie in the 2.25-2.27 $\AA$ range and all bond angles are close to $90^{\circ}$ ). Nevertheless, these $\mathrm{Ce} F$ distances are slightly too short in regards with the + III valence of cerium: an oxidative stress can be considered for the $\mathrm{Ce}^{3+}$ ions in the structure. The substitution of $\mathrm{Ce}^{3+}$ for $\mathrm{K}^{+}$(model B) leads to a significantly larger distortion of the octahedral coordination shell, but with a set of $\mathrm{Ce} F$ distances perfectly respecting the theoretical values for 6 -fold coordination (the cerium calculated valence is 2.99). On the opposite, the substitution of $\mathrm{Ce}^{3+}$ for $\mathrm{K}^{+}$is the less stable situation; it can be argued that this substitution strongly destabilizes the fluorine ion bridging $\mathrm{In}^{3+}$ and $\mathrm{Ce}^{3+}$. This fluorine ion, represented in Figure $\mathrm{S} 2 \mathrm{a}$ in the Supporting Information in the pristine elpasolite, acquires a valence of $1 / 6$ from the $K$ $F$ bond and a valence of $3 / 6$ from the In $F$ bond (completed by four $\mathrm{Rb} F$ bonds with $1 / 12$ valence each). When $\mathrm{Ce}^{3+}$ substitutes for $\mathrm{K}^{+}$, the bridging fluorine ion acquires a valence of $3 / 6$ that is incompatible with the contributions of one In F plus four Rb $\mathrm{F}$ bonds. Hence, significant constraints apply to the bridging Ce-F-In fluorine ion, leading to a significantly higher electronic energy. At last, the substitution of $\mathrm{Ce}^{3+}$ for $\mathrm{Rb}^{+}$is investigated. Model $\mathrm{D}$ leads to a more stable system than model $C$; thus we focus the discussion on model $D$ alone. These last DFT calculations seem to indicate that it is easier to substitute $\mathrm{Ce}^{3+}$ for $\mathrm{Rb}^{+}$than for $\mathrm{K}^{+}$in this elpasolite-type compound. Moreover, an important rearrangement occurs for the ligands around the $\mathrm{Ce}^{3+}$ ion in the $\mathrm{Rb}^{+}$position; the structural relaxation leads to an 8-fold coordination for $\mathrm{Ce}^{3+}$, arising from the 12 -fold cuboctahedral coordination of $\mathrm{Rb}^{+}$ before relaxation (Figure S2 b in the Supporting Information). A reductive stress also takes place on the trivalent cerium, as the calculated valence is found equal to 2.63 .

To sum up, the DFT calculation confirms that cerium cations are preferentially located on the indium site but this creates an oxidation stress on these latter with an equilibrium valence equal to 3.36. A second location of a cerium element could be on the rubidium site. The corresponding coordination polyhedron relaxes to form an 8-fold coordination environment but the calculated valence equal to 2.63 is much lower than the one expected.

\section{Luminescence properties}

As Bridgman technique often shows some segregation of the doping elements along the crystals, all the spectroscopic measurements were performed on a crushed centimeter single crystal in order to obtain an average intensity value of the luminescent centers. The reproducibility of the luminescence phenomenon was easily tested thanks to the large dimension of the synthesized crystals.

The major emission from $\mathrm{Ce}^{3+}$, which is associated with its location in the regular octahedral site of $\mathrm{In}^{3+}$, is represented in Figure $3 \mathrm{a}$ at $10 \mathrm{~K}$ and at room temperature.
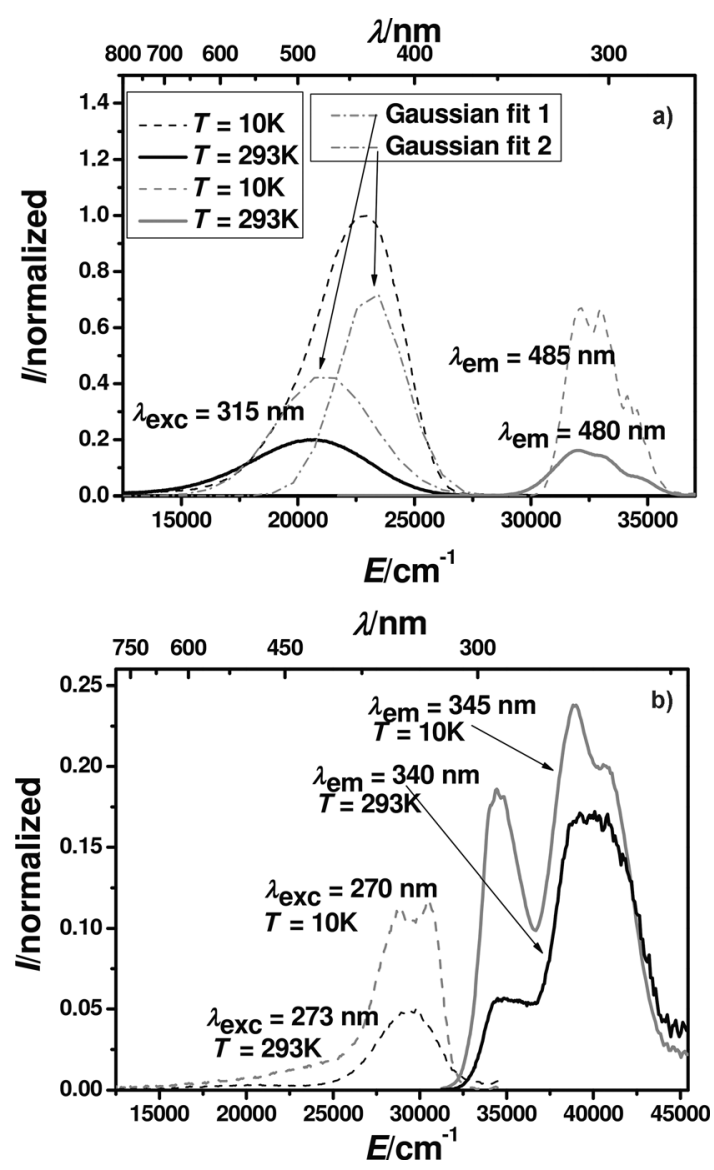

Figure 3. Excitation and emission spectra of the $\mathrm{Ce}^{3+}$ doped $\mathrm{Rb}_{2} \mathrm{~K}_{\ln } \mathrm{F}_{6}$ matrix before irradiation, for a) $\mathrm{Ce}^{3+}$ at the indium site and b) $\mathrm{Ce}^{3+}$ at the rubidium site. 
This observation is in agreement with the DFT calculations. Under excitation at $\lambda=315 \mathrm{~nm}$, a large emission band centered at $\lambda=480 \mathrm{~nm}$ is detected. Fitting with two Gaussian curves indicates an energy difference of $\tilde{v}=1802 \mathrm{~cm}^{-1}$ between the $5 \mathrm{~d}_{\mathrm{T}_{29}}-{ }^{2} \mathrm{~F}_{7 / 2}$ and $5 \mathrm{~d}_{\mathrm{T}_{29}}-{ }^{2} \mathrm{~F}_{5 / 2}$ components. A temperature decrease results in a strong blue shift of the band from $\lambda=480$ to $450 \mathrm{~nm}$. A global quenching of the emission intensity of $76 \%$ is observed between room temperature and $10 \mathrm{~K}$, and the fitting with two Gaussian curves gives an energy difference of $\tilde{v}=1971 \mathrm{~cm}^{-1}$. The corresponding excitation spectra also illustrated in Figure $3 \mathrm{a}$ are composed of the expected $5 d_{\mathrm{T} 2 \mathrm{~g}}$ groups of bands in the energy levels characteristic of the crystal field splitting in an octahedral environment. Location of the $5 d_{E_{g}}$ groups of bands is not accessible on our equipment. The corresponding Stokes shift of $\tilde{v}=10714 \mathrm{~cm}^{-1}$ at room temperature decreases to $\tilde{v}=6343 \mathrm{~cm}^{-1}$ at low temperature. These values correspond to the energy difference between the maximum of the $5 d_{T_{2 g}}$ lowest-energy orbital and the maximum of the $5 \mathrm{~d}_{\mathrm{T}_{29}}-{ }^{2} \mathrm{~F}_{5 / 2}$ highest-energy component.

The positions of the $4 \mathrm{f}-5 \mathrm{~d}$ emission bands of $\mathrm{Ce}$ III in various inorganic halides are listed in Table S5 in the Supporting Information. The central positions of these emissions vary between $\lambda=380$ and $420 \mathrm{~nm}$ and are far from the wavelength $(\lambda=$ $480 \mathrm{~nm}$ ) observed in the indium fluoride elpasolite compound. At room temperature, the cerium(III) emission is significantly red shifted compared with previous examples found in the literature. ${ }^{[25,26]}$ Three parameters can cause such a red shift: 1) a crystal field with, to a first approximation, the energies of the $T_{2 g}$ orbitals all lower than the intensity of the crystal field, 2) the high influence of a Franck-Condon parameter. ${ }^{[27]}$ This value reflects a strong modification of the chemical bonding after absorption of the excitation energy. 3) The average difference between the $5 \mathrm{~d}$ and the $4 \mathrm{f}$ atomic orbitals. The centroid position of these $5 \mathrm{~d}_{\mathrm{T}_{29}}-{ }^{2} \mathrm{~F}_{7 / 2}$ and $5 \mathrm{~d}_{\mathrm{T}_{29}}-{ }^{2} \mathrm{~F}_{5 / 2}$ bands is known to be strongly related to the nature of the cations and the anions constituting the matrix. ${ }^{[6,25]}$ Their influences are argued below. Obviously, when comparing different halides, the crystal field should strongly depend on the ligand (and follows the spectrochemical series: $\left.\mathrm{I}^{-}<\mathrm{Br}^{-}<\mathrm{Cl}^{-}<\mathrm{F}^{-}\right)$. Thus, the highest crystal fields are in fluorides, such as the studied elpasolite, which leads to a lowering of the $t_{2 g}$ bands. The cerium(III) $5 d \rightarrow 4 f$ emission is shifted to low energy. Nevertheless, for the energy emissions reported in the Table S5 in the Supporting Information, this explanation does not seem to be sufficient to fully explain the energy position observed in the Ce-doped $\mathrm{Rb}_{2} \mathrm{KInF}_{6}$. In a series of compounds studied by Birowosuto et al. with different rare-earth elements, when the ratio between the radii of the substituted rare-earth element and the cerium decreases, the energy difference between the $E_{g}$ and $T_{2 g}$ groups of bands increases because of the increase in the crystal field around the $\mathrm{Ce}^{3+}$ cation. ${ }^{[26]}$ The indium(III) ion is significantly smaller than the cerium(III) ion and the corresponding radii ratio is one of the smallest value calculated on the elpasolite materials (Table S5 in the Supporting Information); this difference can also partially explain the very high emission wavelength observed here. However, in the studied matrix, the emission is unexpectedly red shifted relative to the other halide elpasolite-type matrices (Table S5 in the Supporting Information). The energy difference between the absorption and emission bands corresponds to the highest value of Stokes shifts listed. Another possible explanation is that in our elpasolite compound, the average energy level of the $5 \mathrm{~d}$ orbitals (centroid position) is closer to the $4 \mathrm{f}$ energy levels. In the literature, the centroid position of the $5 d$ orbitals is shown to be strongly linked to the polarizability of the metal ligand bond. Aull et al. explained that in elpasolite matrices, the polarization of the $\mathrm{Ce} \mathrm{F}$ bond is greater than that of the $\mathrm{Ce} \mathrm{F}$ bond in $\mathrm{Ce}^{3+}$-doped YLF materials. ${ }^{[25]}$ Hence, in elpasolite systems, the positioning of the cerium/indium, potassium, and fluorine ions along the cell axes is claimed to result in a global drop of the centroid position of the $5 \mathrm{~d}$ energy (stabilization of the $5 \mathrm{~d}_{\mathrm{E}_{g}}$ orbitals and destabilization of the $5 d_{T_{2 g}}$ orbitals). We cannot have access to the $5 d_{E_{9}}$ bands but the $5 d_{T_{2 q}}$ absorption bands peak at the highest energies reported on Table $\mathrm{S} 5$ in the Supporting Information. But this explanation is not totally satisfactory and the global red shift has to result from the three described factors. One final hypothesis can be formulated: the cerium $5 d$ atomic orbitals are stabilized by a metal-metal hybridization involving empty orbitals (in the lowest energy level) of a neighboring metal (such as the 5s5p orbitals of the indium(III)).

When the temperature decreases, in addition to a lower contribution of the Franck-Condon parameter, the large blue shift observed in the emission may reflect a reorganization of the indium octahedral site at low temperature. This structure is known to exhibit two phase transitions at 283 and $263 \mathrm{~K}$ to less symmetric phases. ${ }^{[20]}$ These transitions were observed in the Ce-doped elpasolite through the determination of the $\mathrm{Cp}$ values; values of 0.8576 and $0.5832 \mathrm{~J} \mathrm{~g}^{-1} \mathrm{~K}^{-1}$ were measured for the transitions at 263 and $282 \mathrm{~K}$, respectively. Because of the low proportion of cerium introduced in the matrix, the temperatures of the phase transitions, which perfectly match the values reported by Aleksandrov et al., ${ }^{[20]}$ were unchanged. Although the first coordination sphere around the doping element still exhibits the same coordination sphere, the likely decrease in the symmetry of the site could explain the significant decrease in the crystal field, and thus, the blue shift of the $5 \mathrm{~d} \rightarrow 4 \mathrm{f}$ emission band.

Another much weaker signal was detected in the Ce-doped $\mathrm{Rb}_{2} \mathrm{KInF}_{6}$ materials (Figure $3 \mathrm{~b}$ ), but this signal differs from the data published by Chaminade et al., who attributed the $\lambda=$ $320 \mathrm{~nm}$ band to $\mathrm{Ce}^{3+}$ in the rubidium site and a third band at $\lambda=424 \mathrm{~nm}$ to $\mathrm{Ce}^{3+}$ in the potassium site. ${ }^{[13,28]}$ In the present investigation, a second emission was identified at $\lambda=340 \mathrm{~nm}$ for an excitation at $\lambda=270 \mathrm{~nm}$. The Stokes shift is equal to $\tilde{v}=$ $7630 \mathrm{~cm}^{-1}$. The temperature decrease does not affect the position of the emission band and the thermal quenching is approximately $52 \%$ between $10 \mathrm{~K}$ and room temperature. Several papers report a second fingerprint of trivalent cerium in elpasolite matrices. Birowosuto et al. announced an anomalous emission in bromide materials, ${ }^{[26]}$ and Dorenbos et al., and Bessiere et al. observed this anomalous emission in chloride compounds. ${ }^{[2,30]}$ This band is caused by an unexpected radiative $5 \mathrm{~d}_{\mathrm{E}_{\mathrm{g}}}-{ }^{2} \mathrm{~F}_{5 / 2},{ }^{2} \mathrm{~F}_{7 / 2}$ de-excitation that is rapidly quenched with temperature in favor to the normal $5 \mathrm{~d}_{\mathrm{T}_{29}}$ - 
${ }^{2} \mathrm{~F}_{5 / 2},{ }^{2} \mathrm{~F}_{7 / 2}$ luminescence of $\mathrm{Ce}^{3+}$ in octahedral sites. However, the temperature dependence reported by these authors is not observed in the Ce-doped $\mathrm{Rb}_{2} \mathrm{KInF}_{6}$ compounds. In addition, the two components of the second cerium emission are well resolved in the fluoride matrix. If we consider the argument of Birowosuto et al., the substitution of chlorine by bromine in elpasolite leads to weaker breathing mode vibrational frequencies and results in narrowing of the $5 \mathrm{~d}^{2}{ }^{2} \mathrm{~F}_{5 / 2},{ }^{2} \mathrm{~F}_{7 / 2}$ bands. ${ }^{[26]} \mathrm{Be}-$ cause the decrease in the full width at half maximum is larger for the highest-energy component in the bromide elpasolite, it is consequently easier to discriminate between the two bands. Following the same assumption, we do not expect well-resolved bands for the fluoride elpasolite, in which the phonon energies are higher than those of the chloride elpasolites. A second hypothesis could be the existence of a secondary phase intergrown within the elpasolite during the Bridgman crystal growth process. However, we can discard this hypothesis because the X-ray diffraction pattern of the crushed crystal and the acquisition performed on a single crystal (not given here) tend to confirm the purity of the as-prepared single crystals. A final hypothesis is the location of trivalent cerium in a second environment. This effect has already been reported by Aull et al. in rare-earth-doped $\mathrm{Rb}_{2} \mathrm{NaYF}_{6}$ materials; they concluded that a larger site must exist with a higher coordination than that of the normal blue-green emission. ${ }^{[25]}$ Based on its high energy, we assume that this low-intensity luminescence corresponds to a few proportion of $\mathrm{Ce}^{3+}$ ions in the rubidium cuboctahedral site. For the equivalent coordination sites of $\mathrm{K}^{+}$ and $\mathrm{In}^{3+}$ along a collinear axis, we suggest that if it exists, the luminescence from $\mathrm{Ce}^{3+}$ substituting for $\mathrm{K}^{+}$should be nearly equivalent to that of $\mathrm{Ce}^{3+}$ in the $\mathrm{In}^{3+}$ site. Furthermore, our attribution is well supported by the previous DFT calculations, which showed that the crystal structure is less destabilized when $\mathrm{Ce}^{3+}$ substitutes for $\mathrm{Rb}^{+}$than for $\mathrm{K}^{+}$.

Irradiation of Ce-doped $\mathrm{Rb}_{2} \mathrm{KInF}_{6}$ at $\lambda=315 \mathrm{~nm}$ results in a decrease of the major $\lambda=480 \mathrm{~nm}$ emission in favor of a new red emission at $\lambda=650 \mathrm{~nm}$. The consideration that defects created under irradiation can be at the origin of this anomalous emission was discarded in regard of the analysis of the nondoped matrix. This new emission band was attributed to the $5 s^{1} 5 p^{1} \rightarrow 5 s^{2}$ radiative transition of monovalent indium according to the following equilibrium: $2 \mathrm{Ce}^{3+}+\mathrm{In}^{3+} \rightleftarrows 2 \mathrm{Ce}^{4+}+\mathrm{In}^{+}$. Buñuel et al. fully characterized the spectral distribution and decay time of this irradiation-generated luminescence as a function of temperature. ${ }^{[28]}$ As a reminder, the octahedral environment of $\mathrm{In}^{+}$leads to a splitting of the excited $\mathrm{p}$ levels. The $A_{T}$ and the $A_{X}$ band originate from the Jahn-Teller effect and spin-orbit coupling and present maxima at $\lambda=400$ and $650 \mathrm{~nm}$, respectively. By increasing the temperature. The $X$ minimum of the adiabatic potential energy surface (APES) energy is thermally populated through depopulation of the T minimum of the APES. Only the lowest-energy band is detected at room temperature. The luminescence from $\mathrm{In}^{+}$in its octahedral environment in the $2 \%$ Ce-doped $\mathrm{Rb}_{2} \mathrm{KInF}_{6}$ is illustrated in Figure $4 a, b$, in which the emission and excitation spectra are presented. The two observed emission bands centered at about $\lambda=400$ and $600 \mathrm{~nm}$ can be attributed without any
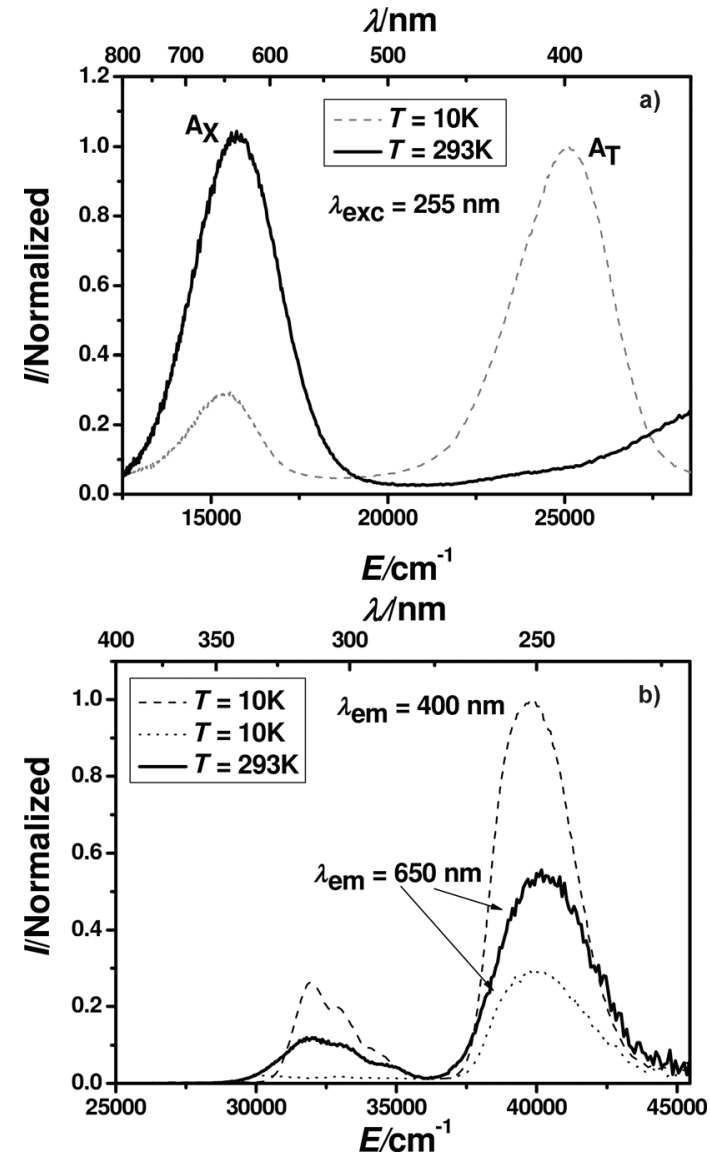

Figure 4. a) Emission and b) excitation spectra of $\ln ^{+}$in $\mathrm{Rb}_{2} \mathrm{KInF}_{6} / \mathrm{Ce}^{3+} 2 \%$ after irradiation.

doubt to the $\mathrm{In}^{+}$ions. Indeed, their characteristic intensity thermal dependence (significant quenching with temperature of the high-energy band) and also the occurrence of an unique absorption band at $\lambda=255 \mathrm{~nm}$, which shows that the two bands get a common origin (they are issued from the same excited level), definitively allow the indexation of these two bands as the $A_{T}$ and $A_{X}$ bands of the $\operatorname{In}^{+}$ions. In addition, cerium luminescence is located between $\lambda=300$ and $325 \mathrm{~nm}$ under a $\lambda=270 \mathrm{~nm}$ excitation beam, which corresponds to the maximum of the excitation spectrum. An excitation at $\lambda=$ $255 \mathrm{~nm}$ leading to a weaker contribution of these emitting centers does not match with the spectral distribution observed in Figure 4.

Because the redox process is expected to be reversible under irradiation, we attempted to cycle the Ce-doped elpasolite material. Crushed single crystals were successively irradiated at $\lambda=315 \mathrm{~nm}$ (excitation at the maximum of the absorption band for $\mathrm{Ce}^{3+}$ in 6-fold coordination) and at $\lambda=255 \mathrm{~nm}$ (excitation at the maximum of the $\ln ^{+}$absorption band) for one hour periods. Figure 5 illustrates the emission spectra for a $\lambda=$ $255 \mathrm{~nm}$ excitation wavelength at room temperature the initial time $\left(t_{0}\right)$, after one hour of irradiation at $\lambda=315 \mathrm{~nm}\left(t_{1}\right)$, and lastly, after one hour of irradiation at $\lambda=255 \mathrm{~nm}\left(t_{2}\right)$.

The resulting graph shows the activation and deactivation of the monovalent indium luminescence; this behavior con- 


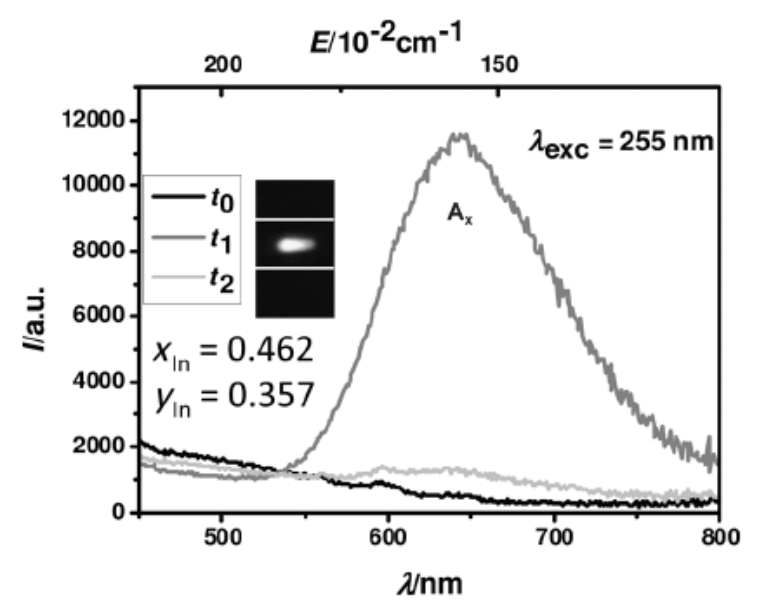

Figure 5. Luminescence properties of $\mathrm{Rb}_{2} \mathrm{KInF}_{6} / \mathrm{Ce}^{3+} 2 \%$ for $t_{0}$ (initial), $t_{1}$ (after $1 \mathrm{~h}$ under $\lambda_{\text {irr }}=315 \mathrm{~nm}$ ), and $t_{2}\left(1 \mathrm{~h}\right.$ under $\lambda_{\text {irr }}=255 \mathrm{~nm}$ ) for the $\mathrm{In}^{+}$ emission spectra. Powder photographs are given for each emission.

firms the reversibility of the reduction-oxidation process under the appropriate irradiation.

This reversible phenomenon is observed on the grown single crystals as well as the departure synthesized powder. Even if the crystal growth process might modify the initial cerium content of the elpasolite phase (potential segregation effect on residual impurities) or might create bulk punctual defects, we can discard the influence of these specific defects in the observed processes.

To the best of our knowledge, this reversible redox phenomenon between cerium and indium under irradiation is unique and most likely results from both the peculiar alternation of cations in the double perovskite structure and the nature of the cations. As mentioned in a previous paragraph, the succession of $\mathrm{K}-\mathrm{F}-\mathrm{In} / \mathrm{Ce}$ along quasi-collinear axes, that is, the positioning of the major portion of the cerium ions in the indium site, is supported by the luminescence studies and the DFT calculations. Hence, under irradiation, the $4 \mathrm{f}$ cerium electron promoted to the $5 \mathrm{~d}$ orbitals relaxes to the $5 \mathrm{~d}_{\mathrm{T}_{2 q}}$ orbitals $\left(5 \mathrm{~d}_{x y} 5 \mathrm{~d}_{x z^{\prime}}\right.$ and $5 d_{y z}$ ), which point between the fluorine ions towards the $\mathrm{In}^{3+}$ ions. One can imagine that the close vicinity of the indium and cerium cations along the cerium $5 \mathrm{~d}_{\mathrm{T}_{2 g}}$ directions (i.e., each of the twelve indium ions in first-neighbor positions to a cerium is strictly located on one of the twelve $d_{T_{2 g}}$ orbital lobes, as shown in Figure S3 in the Supporting Information) favors electron transfer from the excited rare-earth element to the transition metal. Recall that the DFT calculations showed that the $\mathrm{Ce}^{3+}$ ions substituted for $\mathrm{In}^{3+}$ ions are subject to oxidative stress. This stress can also cause the observed interatomic charge transfer. The consecutive reduction of $\operatorname{In}^{3+}$ to $\ln ^{+}$ gives rise to an $n s^{2}$ monovalent cation with a highly polarizable electronic doublet. Thus, "back electron transfer" from indium to cerium under irradiation corresponding to the $\mathrm{In}^{+}$excitation is also highly probable. This electron mobility is also facilitated by the potential metal-metal hybridization. The $2 \mathrm{Ce}^{3+}+\mathrm{ln}^{3+}$ $\rightleftarrows 2 \mathrm{Ce}^{4+}+\mathrm{In}^{+}$redox phenomenon, associated with a drastic change in the luminescence properties of the studied elpasolite compound, can thus be photoinduced from left to right or the reverse, from right to left, by controlling the wavelength of the photoinduction source.

\section{Conclusions}

Despite a low tolerance factor, the Ce-doped $\mathrm{Rb}_{2} \mathrm{KInF}_{6}$ elpasolite compound shows a cubic form at room temperature because of relaxation of the constraints through anionic disordering. Additionally, order-disorder phase transitions take place at low temperature. Based on Rietveld refinements, at room temperature, the fluorine ions were randomly distributed on 1/ 4 of the $96 j$ Wyckoff positions.

DFT calculations showed that the preferential site for cerium substitution is the indium site. Nevertheless, substituting a portion of the $\mathrm{Ce}^{3+}$ for $\mathrm{Rb}^{+}$also seems to be relatively stable, even though this substitution implies a reorganization of the ligands around the introduced $\mathrm{Ce}^{3+}$ ion (an 8-coordinate sphere is predicted by the DFT simulations).

Characterization of the luminescence of the Ce-doped $\mathrm{Rb}_{2} \mathrm{KInF}_{6}$ confirmed that even if the doping element is mainly located in its natural octahedral substitution site (the isovalent indium site), part of this element is present in the $\mathrm{Rb}^{+}$ cuboctahedral polyhedron. Due to the vicinity of cerium and indium cations that should favor a metal-metal hybridization, successive irradiations at $\lambda=315$ and $255 \mathrm{~nm}$ allow reversible oxidation-reduction processes between these elements; these processes are associated with strong spectral modifications of the emission range.

After examination of the crystal irradiated during the studies by Chaminade et al. in $1995,{ }^{[12]}$ we can announce that the resulting irradiated zone is still visible after being maintained at room temperature in a non-UV-exposed environment. Therefore, after a nineteen-year period, the monovalent indium is still stabilized in this cerium-doped fluorinated matrix. This stabilization leads to quite unusual and exciting photosensor properties. For example, such a sensitive material could be used for data storage and is also a good candidate for a photosensor, which would be able to determine the duration of UV exposure in the $\lambda=250-260 / 280-320 \mathrm{~nm}$ ranges after calibration of the system.

\section{Experimental Section}

Synthesis: According to the literature, fluoride elpasolite phases can be synthesized by solid-state routes, ${ }^{[5,31,32]}$ and crystal growth. ${ }^{[13]}$ Two types of crystal growth processes are usually used: the Czochralski or the Bridgman methods. ${ }^{[25]}$ As the photoluminescence described previously, ${ }^{[13]}$ was performed on a $1 \%$ doped materials, we fixed the percentage at a higher concentration ( $2 \%$ of molar substitution at the In site) to increase the effect of the rareearth element introduction on the host lattice.

First, an aqueous solution process allows a polycrystalline powder to be obtained. On the one hand, an acidic aqueous solution was prepared from the dissolution of rubidium carbonate (Aldrich, $99 \%$ ), potassium carbonate (Sigma, $>99.0 \%$ ), and cerium nitrate hexahydrate (Alfa Aesar, $99.99 \%$ ) in nitric acid (68\%); on the other hand, indium oxide (Alfa Aesar, $99.997 \%$ ) was dissolved in hydrofluoric acid (40 mol\% in water). Metallic cations were introduced in 
a stoichiometric proportion in the acid solutions. The solutions were mixed and put on a hot plate with stirring under argon flow until the solvent had evaporated. A solid residue was obtained and was crushed in an agate mortar and mixed with half of its weight of $\mathrm{NH}_{4} \mathrm{HF}_{2}$ (Fluka, $>98.5 \%$ ). The as-prepared powder was put in a furnace and treated at $650^{\circ} \mathrm{C}$ for $1 \mathrm{~h}$ under argon flow. This intermediate step was repeated a second time, that is, the obtained solid was again mixed with half of its weight of $\mathrm{NH}_{4} \mathrm{HF}_{2}$ and was heat treated at the same temperature. This process allows a large amount of powder to be prepared in one unique batch for the crystal growth, which is not possible through the sealed-tube synthesis described by Chaminade et al. ${ }^{[13]}$ A crystal growth process was performed to obtain massive materials for further analysis and to be sure that no trace of impurity could parasite the optical measurements. Single crystals were obtained by the Bridgman crystal growth process. The powder was placed in a platinum crucible under vacuum and was placed in a vertical furnace. The sample was subjected to an initial temperature of $1170^{\circ} \mathrm{C}$, which is higher than the melting temperature $\left(1094^{\circ} \mathrm{C}\right)$; then, the furnace was vertically lifted to decrease the temperature and initiate the crystal growth at a rate of $0.823 \mathrm{mmh}{ }^{1}$. A collection of a few single crystals with various shapes and sizes were obtained (maximum size $1 \times 1 \times 0.8 \mathrm{~cm}^{3}$ ).

Routine XRD measurements performed on a residual polycrystalline phase located in the center of the crucible revealed the presence of a cerium-rich phase $\left(\mathrm{KCeF}_{4}\right)$, which indicates that the final concentration in cerium in the elpasolite phase is inferior to $2 \%$.

$\mathrm{X}$-ray diffraction analysis: Powder X-ray diffraction patterns were collected on a Philips X'Pert MPD X-ray diffractometer with a Bragg Brentano geometry by using $\mathrm{Cu}_{\mathrm{Ka} 1,2}$ radiation $(10<2 \theta<$ $130^{\circ}$, with a step size of $0.02^{\circ}$ and a counting time of $30 \mathrm{~s}$ ). The patterns were refined by using the Rietveld method with the conventional reliability factors. The Fullprof program package was used. $^{[33]}$ The unit cell parameters, the atomic positions, and the Debye Waller factors were refined on the basis of the $F m 3 m$ space group corresponding to the elpasolite structure of composition $\mathrm{Rb}_{2} \mathrm{KInF}_{6} \cdot{ }^{[20]}$

The single-crystal XRD investigation was performed at room temperature on a Bruker AXS Kappa CCD diffractometer and $\mathrm{Mo}_{\mathrm{K \alpha}}$ radiations. A single crystal of suitable size $\left(0.065 \times 0.030 \times 0.010 \mathrm{~mm}^{3}\right)$ was selected. The diffracted intensities were collected by using $\varphi$ $\Omega$ scans in a full sphere of the reciprocal space up to $\theta 32^{\circ}$. The DENZO-Scalepack program suite ${ }^{[34]}$ was used to extract the intensities from the collected frames and to perform a multi-scan absorption correction and scaling of the data. The structure was solved by direct methods in the centrosymmetric space group $\mathrm{Fm} 3 \mathrm{~m}$ by using the SHELXS97 program, ${ }^{[35]}$ and the refinement was made with SHELXL-2014. ${ }^{[3]}$ All the atomic positions were refined, and then anisotropic displacement parameters (ADP) were considered for all the atoms. Crystallographic data, atomic parameters, interatomic distances, and anisotropic displacement parameters are summarized in the Supporting Information.

Photoluminescence analysis: The photoluminescence properties were analyzed by using a Horiba Fluorolog 3 spectrofluorimeter. The excitation spectra were corrected for the variation of the incident flux and the emission spectra were corrected for the transmission of the monochromator and the response of the photomultiplier. The equipment used consists of a $450 \mathrm{~W}$ xenon lamp, an excitation double monochromator, an emission double monochromator and a thermoelectrically cooled photomultiplier tube.

Irradiation was performed on the same equipment. This irradiation corresponds to the application of one hour excitation at $\lambda 315$ and $255 \mathrm{~nm}$ on the materials after opening the excitation monochromator slits at $2 \mathrm{~mm}$.

\section{Acknowledgements}

Lucile Cornu is the holder of the doctoral fellowship supported by the University of Bordeaux. The authors thank the CNRS, the Aquitaine region, the LaPhIA cluster (IDEX Universite de Bordeaux) and the ANR (ANR-2010-BLANC-0820) for financial support. The authors also thank Prof. Michel Pouchard for fruitful discussion.

Keywords: luminescence $\quad \cdot \quad$ materials science
photochromism $\cdot$ redox reactions $\cdot$ sensors

[1] C. Frondel, Am. Mineral. 1948, 32, 84.

[2] L. J. Cross, W. J. Hillebrand, Z. Kristallogr. Mineral. 1887, 12, 495.

[3] G. Meyer, Prog. Solid State Chem. 1982, 14, 141.

[4] P. A. Tanner, Top. Curr. Chem. 2004, 241, 167278.

[5] Y. Xu, S. Carlson, A. Sjodin, R. Norrestam, J. Solid State Chem. 2000, 150, 399.

[6] B. F. Aull, H. P. Jenssen, Phys. Rev. B 1986, 34, 6640.

[7] A. Tressaud, J. Darriet, P. Lagassie, J. Grannec, P. Hagenmuller, Mater. Res. Bull. 1984, 19, 983.

[8] S. S. Pedro, L. P. Sosman, R. B. Barthem, J. C. G. Tedesco, H. N. Bordallo, J. Lumin. 2013, 134, 100.

[9] L. J. Andrews, S. M. Hitelman, Ettore Majorana Int. Sci. Ser. Phys. Sci. 1987, 30, 515

[10] M. Laroche, M. Bettinelli, S. Girard, R. Moncorgé, Chem. Phys. Lett. 1999, $311,167$.

[11] H. Ramanantoanina, W. Urland, F. Cimpoesu, C. Daul, Phys. Chem. Chem. Phys. 2013, 15, 13902

[12] P. Yang, X. Zhou, H. Deng, M. A. Rodriguez, P. L. Feng, E. V. D. van Loef, K. S. Shah, F. P. Doty, IEEE Trans. Nucl. Sci. 2013, 60, 1033.

[13] J. P. Chaminade, A. Garcia, T. Gaewdang, M. Pouchard, J. Grannec, B. Jac quier, Radiat. Eff. Defects Solids 1995, 135, 137.

[14] J. Glodo, E. van Loef, R. Hawrami, W. M. Higgins, A. Churilov, U. Shirwad kar, K. S. Shah, IEEE Trans. Nucl. Sci. 2011, 58, 333.

[15] C. Whitney, C. Stapels, E. Johnson, E. Chapman, G. Alberghini, J. Glodo, K. Shah, J. Christian, Prog. Biomed. Opt. Imaging Proc. SPIE 2011, 7961, 79610 U.

[16] J. P. Chaminade, A. Garcia, Rom. J. Optoelectron. 1997, 5, 15

[17] G. King, P. M. Woodward, J. Mater. Chem. 2010, 20, 5785.

[18] O. V. Kopyshinsky, B. A. Okhrimenko, S. E. Zelensky, B. A. Danilchenko, O. P. Shakhov, J. Phys. Condens. Matter 2003, 15, 3919.

[19] D. Altermatt, I. D. Brown, Int. Union Crystallogr. Crystallogr. Symp. 1985 85, 0108.

[20] K. S. Aleksandrov, S. V. Misyul, M. S. Molokeev, V. N. Voronov, Phys. Solid State 2009, 51, 2505.

[21] I. D. Brown, D. Altermatt, Acta Crystallogr. Sect. B 1985, 41, 244.

[22] G. Kresse, J. Hafner, Phys. Rev. $B$ 1993, 47, RC558.

[23] a) <G. Kresse, J. Furthmüller, Phys. Rev. B 1996, 54, 11169; b) http:// cms.mpi.univie.ac.at/vasp.

[24] J. P. Perdew, K. Burke, M. Ernzerhof, Phys. Rev. Lett. 1996, 77, 3865

[25] B. F. Aull, H. P. Jenssen, Phys. Rev. B 1986, 34, 6647.

[26] M. D. Birowosuto, P. Dorenbos, C. W. E. van Eijk, K. W. Kraemer, H. U. Guedel, J. Phys. Condens. Matter 2006, 18, 6133.

[27] C. W. Struck, W. H. Fonger, J. Lumin. 1975, 10, 1.

[28] M. A. Buñuel, B. Moine, B. Jacquier, A. Garcia, J. P. Chaminade, J. Appl. Phys. 1999, 86, 5045.

[29] P. Dorenbos, E. V. D. van Loef, C. W. E. van Eijk, K. W. Krämer, H. U. Güdel, Phys. Rev. B 2003, 68, 125108.

[30] A. Bessière, A. Lecointre, R. A. Benhamou, E. Suard, G. Wallez, B. Viana, J. Mater. Chem. C 2013, 1, 1252.

[31] I. N. Flerov, M. V. Gorev, K. S. Aleksandrov, A. Tressaud, J. Grannec, M Couzi, Mater. Sci. Eng. R 1998, 24, 81. 
[32] A. Tressaud, J. Fluorine Chem. 2011, 132, 651.

[33] J. Rodriguez Carvajal, Commission on Powder Diffraction (IUCr) News letter, 2001, 26, 12.

[34] Z. Otwinowski, W. Minor in Methods in Enzymology, Macromolecular Crystallography, Part A, Vol. 276 (Eds.: C. W. Carter, R. M. Sweet), Academ ic Press, London, 1997.

[35] SHELXS 97, G. M. Sheldrick, University of Göttingen (Germany), 1997.
[36] SHELXL 2014/7, G. M. Sheldrick, University of Göttingen (Germany), 2014. 